- Notes -

\title{
A Microflow-Extraction System Using Double Tubes Having Different Inner Diameters in Tube Radial Distribution Phenomenon
}

\author{
Katsuya UNESAKI, ${ }^{1}$ Masahiko HASHIMOTO ${ }^{1}$ and Kazuhiko TSUKAGOSHI ${ }^{1,2} *$ \\ ${ }^{1}$ Department of Chemical Engineering and Materials Science, Faculty of Science and Engineering, \\ Doshisha University, Kyotanabe, Kyoto 610-0321, Japan \\ ${ }^{2}$ Tube Radial Distribution Phenomenon Research Center, Doshisha University, Kyotanabe, Kyoto \\ 610-0321, Japan
}

(Received March 17, 2014; Accepted May, 12)

When homogeneous solutions that feature two-phase separation properties, such as a non-ionic surfactant aqueous solution, are fed into an open-tubular capillary tube, the solvent molecules are radially distributed into the tube, generating inner and outer phases. This is called "tube radial distribution phenomenon" (TRDP). In this study, a novel microflow-extraction system was proposed using double capillary tubes having different inner diameters in the TRDP. The tubes were fused-silica capillary tubes with a 75 and 250 $\mu \mathrm{m}$ inner diameter; the smaller tube was inserted into the larger one through a T-type joint. A homogeneous aqueous solution containing $12 \mathrm{wt} \%$ Triton X-100 as a non-ionic surfactant and $2.4 \mathrm{M}$ potassium chloride was fed into the larger tube at a flow rate of $20 \mu \mathrm{L} \mathrm{min}^{-1}$, where the tube was maintained at a temperature of $34^{\circ} \mathrm{C}$. The homogeneous aqueous solution changed to a heterogeneous solution with two phases in the tube; the surfactant-rich phase was generated in the middle of the tube as an inner phase, while the aqueous phase containing a little of the surfactant was formed near the tube wall as an outer phase. In the TRDP Rhodamine B dissolved in the homogeneous solution was distributed into the inner surfactant-rich phase. The distributed Rhodamine B (red color) was observed with a bright-field microscope-CCD camera system. In the present microflow-extraction system, taking advantage of the TRDP, the inner phase containing Rhodamine B flowed inside the smaller tube, while the outer phase flowed outside the tip of the smaller tube into the larger tube, which was made using double capillary tubes having different inner diameters. This observation showed that Rhodamine B dissolved in the homogeneous solution was separated or extracted into the inner surfactant-rich phase through the double capillary tubes based on the specific microfluidic behavior of the TRDP.

\section{Introduction}

The development of micro-total analysis systems that include microfluidic device technology is an interesting aspect of analytical chemistry [1]. Microfluidics exhibit various types of fluidic behavior of solvents in a microspace. This microfluidic behavior has been studied by varying the flow rate of the solvents by using aqueous-organic solvent mixtures and by introducing specific obstacles into the microspace [2-4]. The fluidic behavior of the solvents in the microspace is related to the mixing, separation, diffusion, and reaction of the solutes. Extraction is, generally, one of the most useful and effective 
separation methods; however, recently, microflow solvent extraction systems have been investigated by using a liquid-liquid interface between an aqueous and organic solvent solution in the microspace [5-7]. Microflow solvent extraction has several advantages over conventional macroscale solvent extraction. For example, the microflow system has a larger surface/interface area per unit volume than that of the conventional macroscale system and the increase in the interface area provides enhancement of extraction efficiency [5].

We reported that, when homogeneous solutions that feature a two-phase separation property, such as a water-acetonitrile-ethyl acetate mixed solvent solution and non-ionic surfactant aqueous solutions, are fed into a microspace, such as microchannels in a microchip or capillary tubes, the solvent molecules are radially distributed in the microspace under laminar flow conditions. This is called the "tube radial distribution phenomenon" (TRDP) [8-11]. For example, in the TRDP with a non-ionic surfactant aqueous solution, through the two-phase separation property, a surfactant-rich phase is formed around the center of the microspace as an inner phase, while the aqueous, or almost surfactant-free, phase is formed near the inner wall as an outer phase [12]. The TRDP creates a specific liquid-liquid interface that is not static but kinetic in the microspace, providing an inner and outer phase.

In this study, a novel microflow-extraction system was presented by using double capillary tubes having different inner diameters: smaller and larger ones [11]. A homogeneous aqueous solution containing a non-ionic surfactant was fed into the larger tube, leading to the TRDP that created the inner surfactant-rich phase and the outer almost surfactant-free aqueous phase. At the tip of the smaller tube in the larger one, the inner phase flowed inside the smaller tube, while, the outer phase flowed outside the smaller tube. The specific flow showed the possibility that substances dissolved in the homogeneous solution were extracted into the inner phase, or surfactant-rich phase, through the double capillary tubes, based on the TRDP.

\section{Experimental}

\subsection{Reagents and materials}

Water was purified with an Elix 3 UV system (Millipore Co., Billerica, MA). All reagents used were obtained commercially and were of analytical grade. Triton X-100 was purchased from Nacalai Tesque, Inc. (Kyoto, Japan). Rhodamine B and potassium chloride $(\mathrm{KCl})$ were purchased from Wako Pure Chemical Industries, Ltd. (Osaka, Japan). Fused-silica capillary tubes (75 $\mu \mathrm{m}$ inner diameter and $150 \mu \mathrm{m}$ outer diameter; $250 \mu \mathrm{m}$ inner diameter and $350 \mu \mathrm{m}$ outer diameter) were purchased from GL Science (Tokyo, Japan).

\subsection{Apparatus and procedures}

A schematic diagram of the present microflow extraction system using double capillary tubes having different inner diameters is shown in Figure 1. The smaller tube ( $75 \mu \mathrm{m}$ i.d.) was inserted into the larger one $(250 \mu \mathrm{m}$ i.d., $120 \mathrm{~cm}$ length) through a T-type joint. A homogeneous aqueous solution containing 12 wt \% Triton X-100 and 2.4 M KCl was fed into the larger tube at a flow rate of $20 \mu \mathrm{L} \mathrm{min}^{-1}$, and the tube was maintained at a temperature of $34^{\circ} \mathrm{C}$ with a thermo-heater (Thermo Plate MATS-555RO; Tokai Hit Co., Shizuoka, Japan). The homogeneous aqueous solution changed to a heterogeneous solution with two phases in the capillary tube. The radial distribution of the surfactant was observed through Rhodamine B dissolved 
in the homogeneous aqueous solution with a bright-field microscope-CCD camera system that was comprised of a microscope (BX51; Olympus, Tokyo, Japan) and a CCD camera (JK-TU53H; Toshiba, Tokyo, Japan).

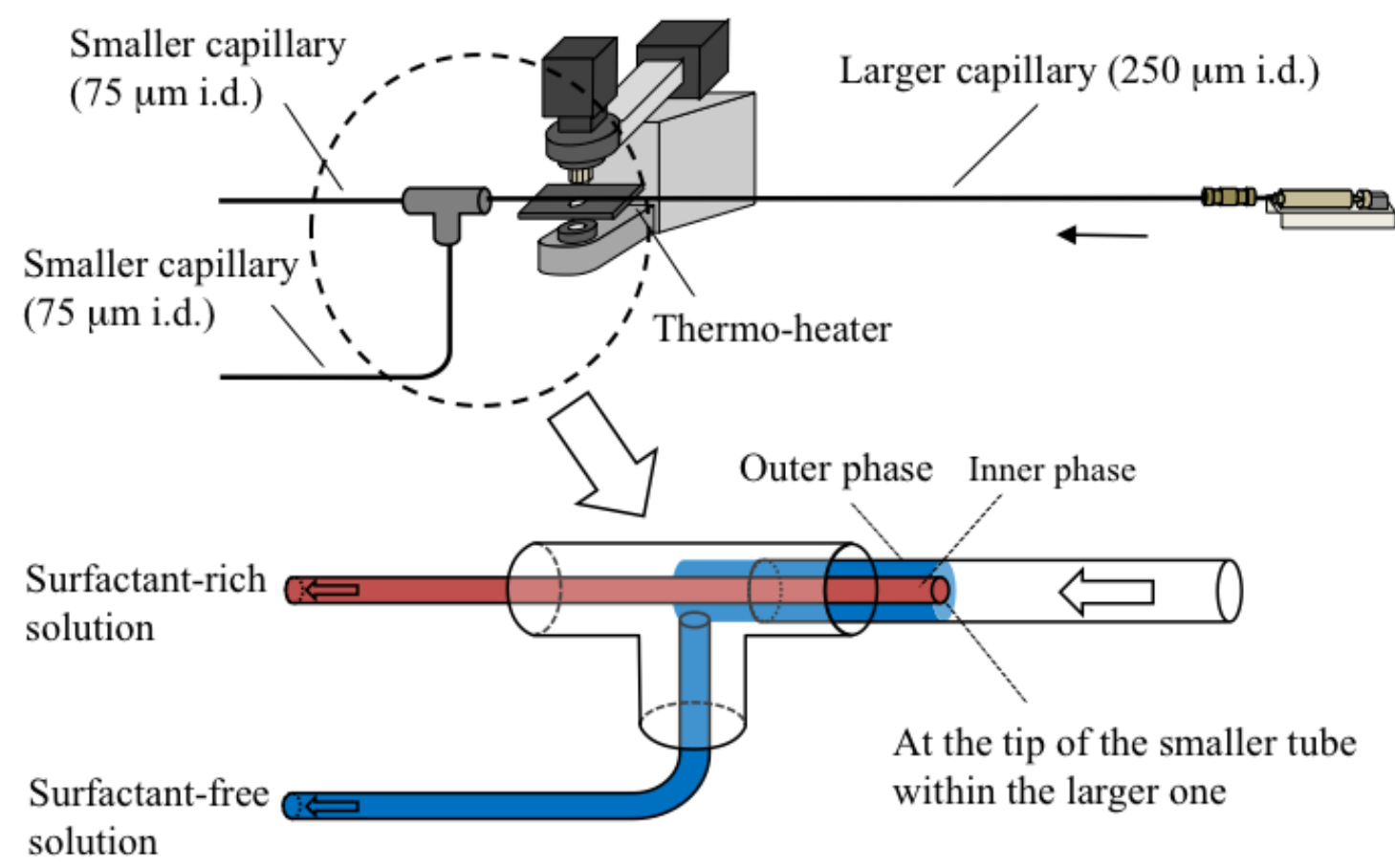

Figure 1. Schematic diagram of the microflow extraction system using double capillary tubes having different inner diameters.

\section{Results and Discussion}

\subsection{Two-phase separation in a batch vessel}

Homogeneous aqueous solutions of specific non-ionic surfactants separate into two distinct phases in a batch vessel when heated above a certain temperature (i.e., the cloud point); a temperature-induced phase separation occurs [13-15]. One phase behaves as a concentrated surfactant solution containing considerable amounts of water (surfactant-rich phase), whereas the other phase is an almost surfactant-free aqueous solution. The hydrogen bond between the oxygen atom of an ether bond in a non-ionic surfactant and a water molecule leads to good solubility of the surfactant in water. However, at temperatures above the cloud point the hydrogen bond is broken, leading to the separation of the surfactant-rich and aqueous phases. In addition, the bond breaking or dehydration increases in the presence of salts. Hydrophobic compounds dissolved in the homogeneous aqueous solution are extracted into the surfactant-rich phase, whereas hydrophilic compounds remain in the aqueous phase.

The cloud points for an aqueous solution containing $2 \mathrm{wt} \%$ Triton X-100 as a non-ionic surfactant were determined for various concentrations of $\mathrm{KCl}, 0-3.0 \mathrm{M}$. The obtained data, or phase diagram, is shown in Figure 2. The homogeneous and transparent solution at $25^{\circ} \mathrm{C}$ changed through the cloud point into a heterogeneous solution of two phases: an upper surfactant-rich suspension phase and an aqueous 
lower phase. The cloud points gradually decreased from $65^{\circ} \mathrm{C}$ to $30^{\circ} \mathrm{C}$ with an increasing $\mathrm{KCl}$ concentration from 0 to $3.0 \mathrm{M}$ as shown in Figure 2.

Also, cloud points of an aqueous solution containing $2.4 \mathrm{M} \mathrm{KCl}$ were examined with various concentrations of Triton X-100, 0-30 wt \%. The homogeneous solution at $25^{\circ} \mathrm{C}$ changed through the clouding point to a heterogeneous solution of two phases, a surfactant-rich phase and an aqueous phase, at $34^{\circ} \mathrm{C}$ for all concentrations of Triton X-100 (data not shown).

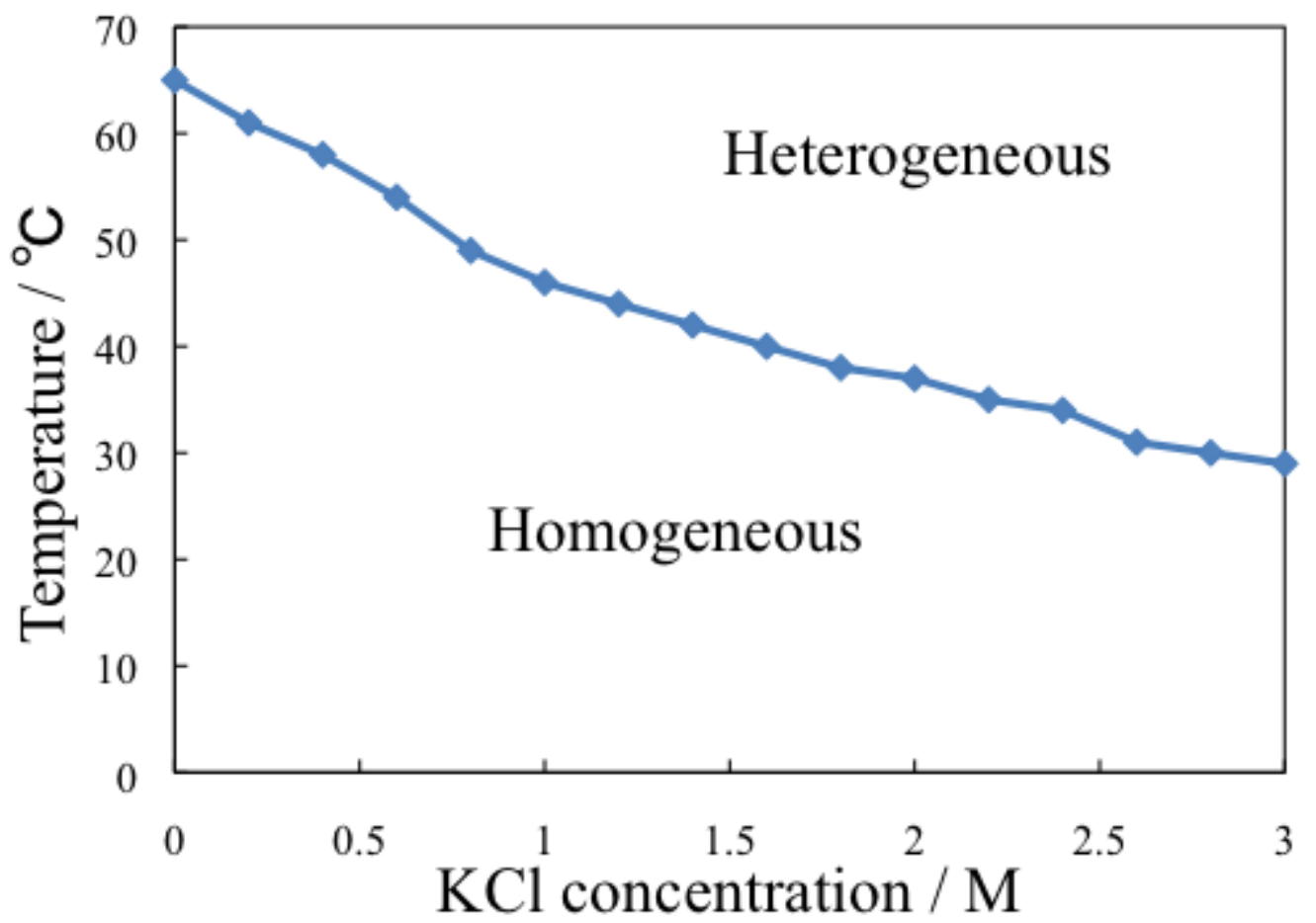

Figure 2. Phase diagram of aqueous solutions containing Triton X-100 and KCl. 2 wt\% Triton X-100 and various concentrations of $\mathrm{KCl}$. The solubility curve between the homogeneous and heterogeneous solutions is shown with the cloud points.

\subsection{TRDP observation}

Homogeneous aqueous solutions containing Triton X-100 (2, 12, 22, and $30 \mathrm{wt} \%)$ and $2.4 \mathrm{M} \mathrm{KCl}$ possessed a cloud point of $34^{\circ} \mathrm{C}$, as described above. The homogeneous solutions that included $5 \mathrm{mM}$ Rhodamine B were fed into the capillary tube $\left(250 \mu \mathrm{m}\right.$ i. d.) at a flow rate of $20 \mu \mathrm{L} \mathrm{min}{ }^{-1}$ under laminar flow conditions, where the tube was maintained at temperatures of $25^{\circ} \mathrm{C}$ or $34^{\circ} \mathrm{C}$. The bright-field photographs of the aqueous solution were taken with the microscope-CCD camera system. Non-TRDP behavior (homogeneous) was observed at $25^{\circ} \mathrm{C}$ and the TRDP was created at $34^{\circ} \mathrm{C}$ for all aqueous solutions.

Typical photographs obtained with the aqueous solution containing 12 wt\% Triton X-100 and 2.4 $\mathrm{M} \mathrm{KCl}$ are shown in Figure 3. The homogeneous red solution as a result of Rhodamine B was observed at a temperature of $25^{\circ} \mathrm{C}$, which was less than the clouding point. That is, the distribution of the surfactant in 
the tube did not occur at a temperature of $25^{\circ} \mathrm{C}$ (non-TRDP). At a temperature of $34^{\circ} \mathrm{C}$, the solution phase containing the surfactant was generated in the middle of the tube as an inner phase (the surfactant-rich phase) and a solution phase containing little of the surfactant was formed near the inner wall as an outer phase (the aqueous phase). Rhodamine B dissolved in the feed solution was distributed to the surfactant-rich phase (red color). The homogeneous aqueous solution containing $12 \mathrm{wt} \%$ Triton X-100 and $2.4 \mathrm{M} \mathrm{KCl}$ with a clouding point of $34^{\circ} \mathrm{C}$ clearly brought about phase transformation to a heterogeneous solution with a surfactant-rich phase and an aqueous phase through the tube, the temperature of which was controlled at $34^{\circ} \mathrm{C}$ (TRDP).
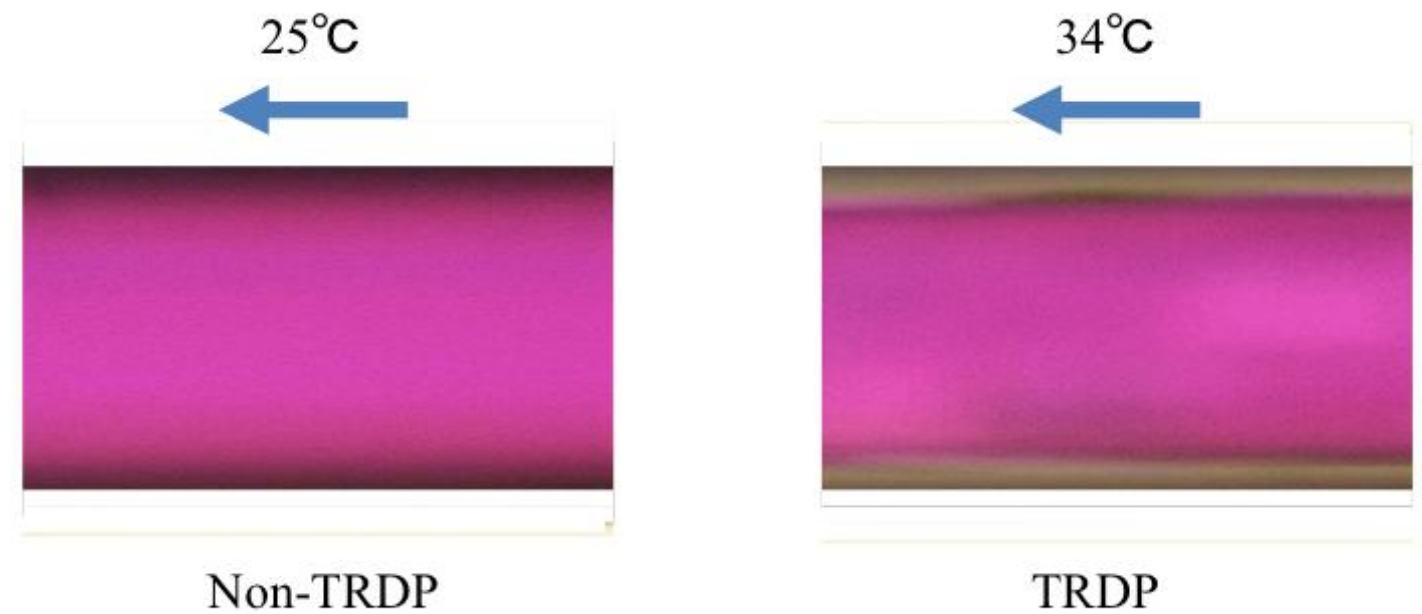

Figure 3. Bright-field photographs in the larger capillary tube $\left(250 \mu \mathrm{m}\right.$ i.d.) at $25^{\circ} \mathrm{C}$ and $34^{\circ} \mathrm{C}$. The homogeneous aqueous solution containing $12 \mathrm{wt} \%$ Triton $\mathrm{X}-100,2.4 \mathrm{M} \mathrm{KCl}$, and $5 \mathrm{mM}$ Rhodamine B was fed at a flow rate of $20 \mu \mathrm{L} \mathrm{min}^{-1}$ into the larger capillary tube.

\subsection{Microflow extraction with TRDP}

The homogeneous aqueous solution containing $12 \mathrm{wt} \%$ Triton X-100, $2.4 \mathrm{M} \mathrm{KCl}$, and $5 \mathrm{mM}$ Rhodamine B was fed at a flow rate of $20 \mu \mathrm{L} \mathrm{min}^{-1}$ into the larger capillary tube. The aqueous solution, containing $12 \mathrm{wt} \%$ Triton X-100, was selected mainly because of the volume ratio of the two phases and because the viscosity of the solution was such that it could be easily delivered into the capillary tube. The photographs obtained at the tip of the smaller tube within the larger one at $25^{\circ} \mathrm{C}$ and $34^{\circ} \mathrm{C}$ are shown in Figure 4. At $25^{\circ} \mathrm{C}$, the homogeneous red solution resulting from Rhodamine $\mathrm{B}$ was fed without any specific fluidic behavior. On the other hand, notable and specific fluidic behavior was observed on the tip of the smaller tube at $34^{\circ} \mathrm{C}$. The inner phase (red color) containing Rhodamine B flowed inside the smaller tube, while the outer phase flowed outside the small tube, as shown in Figure 4. This observation implies that Rhodamine B dissolved in the homogeneous solution was separated or extracted into the inner phase (surfactant-rich phase) through the double capillary tubes based on the specific microfluidic behavior, TRDP. 

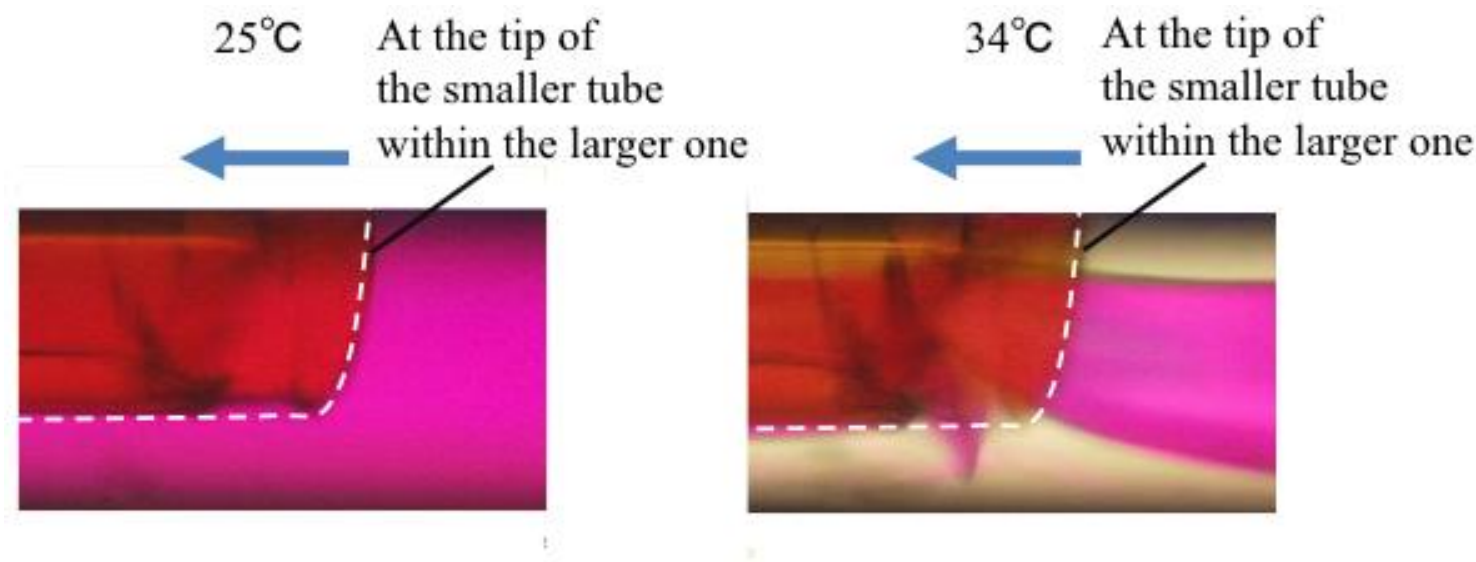

Non-TRDP

TRDP

Figure 4. Bright-field photographs at the tip of the smaller capillary tube ( $75 \mu \mathrm{m}$ i.d.) within the larger tube $\left(250 \mu \mathrm{m}\right.$ i.d.) at $25^{\circ} \mathrm{C}$ and $34^{\circ} \mathrm{C}$. The homogeneous aqueous solution containing $12 \mathrm{wt} \%$ Triton $\mathrm{X}-100,2.4$ $\mathrm{M} \mathrm{KCl}$, and $5 \mathrm{mM}$ Rhodamine B was fed at a flow rate of $20 \mu \mathrm{L} \mathrm{min}^{-1}$ into the larger capillary tube.

\section{Conclusions}

A novel microflow extraction process was demonstrated using double capillary tubes having different inner diameters, a smaller and larger tube, in the specific microfluidic behavior of TRDP. A homogeneous aqueous solution containing Triton $\mathrm{X}-100$ as a non-ionic surfactant and $\mathrm{KCl}$ was fed into the larger tube, where the tube was maintained at the cloud point temperature. The homogeneous aqueous solution changed to a heterogeneous solution of two phases in the tube: the surfactant-rich inner phase and nearly surfactant-free outer phase (TRDP). Rhodamine B dissolved in the homogeneous solution was distributed into the surfactant-rich inner phase in the TRDP. Rhodamine B was extracted into the surfactant-rich phase through the double capillary tubes based on the specific microfluidic behavior of the TRDP.

\section{Acknowledgement}

This work was supported by a Grant-in-Aid for Scientific Research (C) from the Ministry of Education, Culture, Sports, Science, and Technology (MEXT), Japan. This work was also supported by the Advanced Study for Integrated Particle Science, and Technology, Strategic Development of Research Infrastructure for Private Universities, the MEXT, Japan.

\section{References}

1) D. R. Reyes, D. Iossifidis, P. A. Auroux, A. Manz, Anal. Chem., 74, 2623-2636 (2002).

2) A. Hibara, M. Tokeshi, K. Uchiyama, H. Hisamoto, T. Kitamori, Anal. Sci., 17, 89-93 (2001).

3) N. Kaji, Y. Okamoto, M. Tokeshi, Y. Baba, Chem. Soc. Rev., 39, 948-956 (2010).

4) H. Nakamura, Y. Yamaguchi, M. Miyazaki, H. Maeda, M. Uehara, Chem. Commun., 23, 2844-2845 (2002).

5) M. Maeki, Y. Hatanaka, K. Yamashita, M. Miyazaki, K. Ohto, Solvent Extr. Res. Dev., Jpn., 21, 77-82 (2014). 
6) ZX. Cai, Q. Fang, HW. Chen, ZI. Fang, Anal. Chem. Acta, 556, 151-1566 (2006).

7) A. Aota, M. Nonaka, A. Hibara, T. Kitamori, Angew. Chem. Int. Ed, 46, 878-880 (2007).

8) N. Jinno, M. Murakami, K. Mizohata, M. Hashimoto, K. Tsukagosi, Analyst, 135, 927932 (2011).

9) M. Murakami, N. Jinno, M. Hashimoto, K. Tsukagoshi, Anal. Sci., 27, $793-798$ (2011).

10) S. Fujinaga, K. Unesaki, S. Negi, M. Hashimoto, K. Tsukagoshi, Anal. Methods, 4, 3884-3890 (2012).

11) K. Tsukagoshi, Anal. Sci., 30, 65-73 (2014), and references cited therein.

12) N. Jinno, K. Unesaki, M. Hashimoto, K. Tsukagoshi, J. Anal. Chem., 68, 1197-1202 (2013).

13) K. Fujinaga, Anal. Sci., 9, 479-482 (1993).

14) T. Saitoh, H. Tani, T. Kamidate, H. Watanabe, Trends Anal. Chem., 14, 213-217 (1995).

15) P. I. Trindade, M. M. Diogo, D. M. F. Prazeres, C. J. Marcos, J. Chromatogr. A, 1082, 176-184 (2005). 